\title{
Article \\ Geometric Design and Prototyping of a (2-RRU)-URR Parallel Mechanism for Thumb Rehabilitation Therapy
}

\author{
Woo-hyeok Choi *(D) and Yukio Takeda (D) \\ Department of Mechanical Engineering, Tokyo Institute of Technology, 2-12-1 Ookayama, Meguro-ku, \\ Tokyo 152-8552, Japan; takeda.y.aa@m.titech.ac.jp \\ * Correspondence: choi.w.ac@m.titech.ac.jp; Tel.: +81-3-5734-2177
}

Citation: Choi, W.-h.; Takeda, Y. Geometric Design and Prototyping of a (2-RRU)-URR Parallel Mechanism for Thumb Rehabilitation Therapy. Machines 2021, 9, 50. http://doi.org/ 10.3390/machines 9030050

Academic Editors:Domenico Mundo and Vincenzo Niola

Received: 27 January 2021

Accepted: 22 February 2021

Published: 26 February 2021

Publisher's Note: MDPI stays neutral with regard to jurisdictional claims in published maps and institutional affiliations.

Copyright: (C) 2021 by the authors. Licensee MDPI, Basel, Switzerland. This article is an open access article distributed under the terms and conditions of the Creative Commons Attribution (CC BY) license (https:// creativecommons.org/licenses/by/ $4.0 /)$.

\begin{abstract}
In this paper, the geometrical design of a (2-RRU)-URR (where R and U stand for the revolute and universal joints, respectively) parallel mechanism was demonstrated for thumb rehabilitation therapy. This paper consists of two parts: the design procedure for the development of a thumb rehabilitation device and the user experiment with the prototype. Because the hand generally has a limited working area, the design of the hand attachment parts and the placement of the actuators requires careful consideration of the various factors. Along with the kinematic requirements of the device, the interaction between the mechanism and the fingers must be considered. The proposed mechanism has three actuators placed in the hand attachment. When the mechanism is attached to the hand, there is the possibility of collisions between the fingers of the user and the mechanism. Two design candidates were devised while considering the limited working area of the hand and the need to avoid collisions. Due to the dependency of the workspace on the placement of the actuators, a comparison of the workspace of the two candidate designs and the target workspace was carried out. The target workspace was determined through the use of thumb trajectory measurement data. A prototype was manufactured using 3D printed plastic and aluminum materials. To confirm the practical performance of the prototype, user experiments were conducted in which a comparison between the thumb measurement data and the controlled trajectory of each person was done. Motion in two directions, specifically, adduction-abduction and flexion-extension were performed. The results showed that the controlled trajectory of flexion-extension were closely matched to the thumb measurement trajectory. Finally, the experimental results are discussed.
\end{abstract}

Keywords: mechanism design; thumb rehabilitation; parallel mechanism; ergonomics

\section{Introduction}

Thumbs are a critical element of human hands as they facilitate dexterity when humans grasp small objects. In general, combination movements involving the thumb and other fingers cover $40 \%$ of complete hand movements [1]. Consequently, patients who lose thumb movement functionality find it challenging to perform daily life activities. Owing to their functional importance, the rehabilitation and grafting of thumbs are of high priority. As a type of therapy, physical rehabilitation aims to recover the movement function of human body parts. Specifically, an external force is used to stimulate the body motor systems of impaired parts. Usually, the therapist provides the required external force during therapy, the magnitude of which depends on the degree of paresis of the patient. The rehabilitation effect depends on various factors such as the detection time of the disease, attitude of the patient, and engagement of the therapist. In particular, the therapist's engagement with the patient is related to the number of therapy sessions. However, the treatment time is very limited even if the therapists can provide the patients with complete engagement (Only 5.2\% in the two weeks [2].) In addition, the number of young therapists is decreasing due to the aging of society [3]. Therefore, measures to address the lack of therapists are being widely researched in various domains. As a potential solution, rehabilitation robots 
have been introduced. Because robots do not experience fatigue, rehabilitation robots can provide continuous and consistent external force. In general, the human thumb consists of three joints, namely, the interphalangeal (IP) joint, metacarpophalangeal (MP) joint, and carpometacarpal (CMC) joint, as shown in Figure 1. When the hand is in the anatomically resting position, the thumb MP joint axis is oriented approximately $90^{\circ}$ to the MP axis of the other finger [4]. The thumb exhibits five movements, namely, abduction, adduction, flexion, extension, and opposition as shown in Figure 2. Abduction refers to the movement of the thumb moving away from the palm from its resting position. In detail, two kinds of abduction exist regarding the thumb direction [5]. The palmar abduction is the thumb movement wherein the thumb moves perpendicular to the palm. The radial abduction is the movement wherein the thumb extends away from the palm's surface. In this paper, abduction refers to radial abduction. Adduction is the movement of the thumb making it aligned with the palm plane. Flexion is the bending movement of the thumb from the resting position to the surface of the palm. Extension is the movement of the thumb from the flexion state to the resting position. Opposition is the movement of the thumb's tip to the tip of the other fingers. Because the role of the CMC joint is the same in opposition and flexion, the movements are jointly termed as flexion in this work. In terms of the continuous movements such as adduction-abduction (AA) and flexion-extension (FE), the role of the AA is to control the position of the thumb, and FE serves to grasp an object. These roles can be easily visualized considering the situation of catching a ball in one hand.

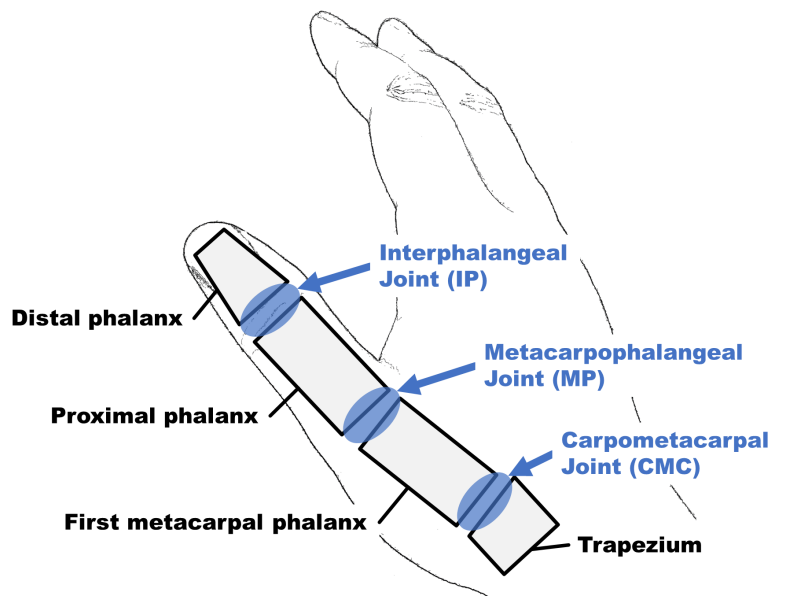

Figure 1. Joints and bones of the thumb.

The thumb's CMC joint exhibits a biconcave-convex surface between the first MP phalanx and trapezium. This joint is usually known as a saddle joint owing to its saddlelike shape. Owing to this shape, an additional sliding movement occurs when the thumb moves. This sliding movement is not independent of the thumb movements; and thus, although the number of degrees of freedom (DOF) of the thumb is 2, the sliding motion has an effect of the position of the thumb. Previous studies of rehabilitation robots, exoskeleton type, and end-effector type, were reported [6]. Specifically, in the exoskeleton type, the robot was attached to the human body, and the actuator axis was aligned to the axis of the human joints sequentially. The end-effector type is a mechanical design that connects the mechanism's end-effector to the fingertip, but the base of the device is far from the body.

In previous studies, the most frequently used type of thumb rehabilitation robot was the exoskeleton. These existing researches pertaining to the thumb can be divided into three categories depending on the consideration of the thumb DOF [7-10]. In the first approach, one DOF is fixed, and the actuator controls the other. This mechanism is primarily designed to control the FE movement, which, as mentioned previously, is more important than the AA movement. However, this method cannot be used to provide the complete range of DOF of the $\mathrm{CMC}$ joint. In the second approach, the actuator controls one $\mathrm{DOF}$, and the other DOF is implemented through a passive joint. The complete range of 
DOF of the CMC joint can be considered. However, most rehabilitation users are patients with paresis. In general, the impaired limbs easily exhibit flexor motions (bending), and the patient cannot control their fingers well. Therefore, the passive DOF is fixed in the direction of the paralyzed side. Due to this, the motion of the controlled DOF may be disrupted by the paralysis of the passive DOF. Because of the disruption caused by the passive DOF, the range of DOF may decrease or be completely blocked. Considering this situation, this method may not be able to provide adequate control for the whole thumb movement. Therefore, this design is not suitable to be applied for rehabilitation purposed. In the third approach, the two DOF are controlled by two actuators. Each actuator adapts one DOF. However, the aforementioned sliding movements are not considered.
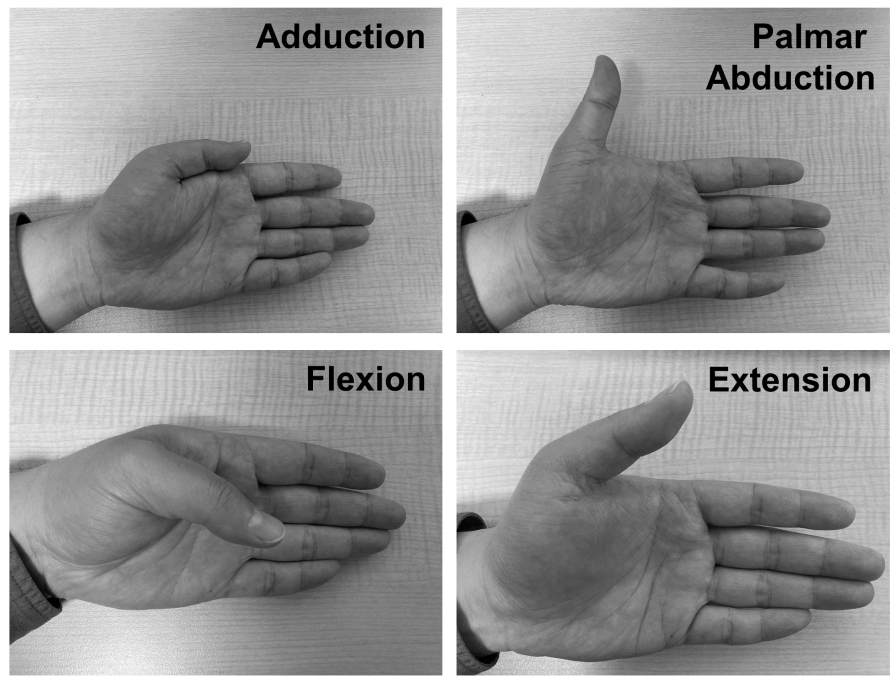
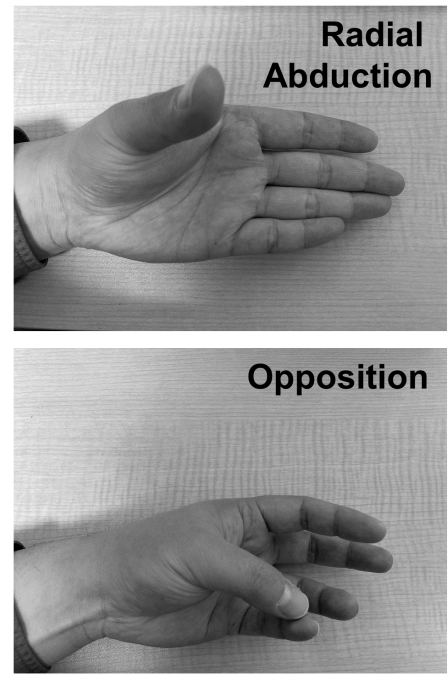

Figure 2. The thumb movements: Adduction, Palmar Abduction, Radial Abduction, Flexion, Extension, and Opposition.

Furthermore, the exoskeleton type has an issue called misalignment. Misalignment occurs when there is a mismatch between the axes of the human joint and the kinematic joint. In this situation, the actuator of the rehabilitation robot may provide an uncontrolled force to the user's finger, leading to a high possibility of injury. It is a very critical problem for the safety of the users. As a solution to this issue, mechanical linkage designs (redundant linkage [11,12], remote center of rotation mechanisms [13-16], and serial linkage mechanisms [8-10]) were reported. However, these linkages only considered the other fingers. Therefore, these designs cannot be considered for thumb rehabilitation due to the difference in DOF and CMC joint complexity. Even if the mechanism would be designed to suit of the DOF of the CMC joint, there is a possibility that the complexity of the mechanism would increase significantly because of the narrow space of the hand.

On the other hand, the end-effector type was designed such that its attachment part is connected to the tip of the finger, and the mechanism's joints do not control each finger joint angle. For this reason, the consideration of misalignment is not necessary because this mechanism's design does not align its kinematic joints with the finger joints $[6,17]$. This mechanism design provides several advantages. One such advantage is that this mechanism can easily adapt to the different sizes of various patient hands. Furthermore, the attachment part does not have a heavy weight because its base part, where the heaviest part of the mechanism is located, is usually installed on a table or stand. However, this type of mechanism results in a lower mobility than the exoskeleton type. Furthermore, the attaching point is mostly the distal part of the finger, and sensitive control for the individual finger joint angles is difficult.

In summary, the sliding motion of CMC joint should be considered for providing accurate finger movement to develop a thumb rehabilitation robot. Furthermore, the mechanism is required to be designed so that it does not exert an unexpected force in 
order to maintain user safety. A hybrid mechanism, combined with the advantage of the exoskeleton and end-effector types, is proposed as an idea for the mechanism. In detail, the design is considered so that multiple linkages connecting only to the attachment point are designed, similar to an end-effector type mechanism, and the base parts of the mechanism are designed to be attached to the hand like an exoskeleton type.

Moreover, it is necessary to perform an ergonomic design to ensure that the patient's hand does not feel uncomfortable and adapts their hand size. Therefore, some parts would be better to be designed by many curve-linear forms, complex shapes, and tiny sizes. Thanks to the recent progress of the Additive Manufacturing (AM), fabrications of these parts are enabled at low cost. For this reason, some parts will be designed to consider the manufacturing by AM in this research.

Based on this concept, a (2-RRU)-URR parallel mechanism is proposed, as shown in Figure 3 [18-20]. This mechanism is an overconstrained mechanism, with three DOF. The mechanism can output two rotational DOF and one translational DOF. The proposed mechanism consists of three chains, the output link, and the base. Structurally, the first and second chains are connected with the output link through a universal joint. The third chain is connected with the output link through a revolute joint. In previous work [18-20], we reported the basic kinematics such as mobility, displacement, workspace analysis, and static analysis for calculating the actuation torque against the external load on the output link. In detail, a numerical example was used for calculating each analysis for the confirmation of the kinematics. Furthermore, the static analysis of the proposed mechanism had been confirmed through an experiment with the experimental apparatus. Through the results of the previous works, the kinematic characteristics were confirmed for the design of the mechanism. However, because the proposed mechanism being designed for thumb rehabilitation, the mechanism design should take into consideration the human measurement data, which provides kinesiological/anatomical information. Moreover, the proposed mechanism is designed to be attached to the hand. To realize the implementation of the proposed mechanism, user experiments are necessary.

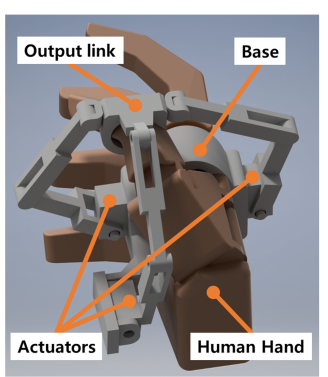

(a)

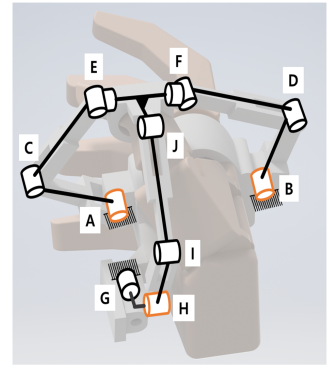

(b)

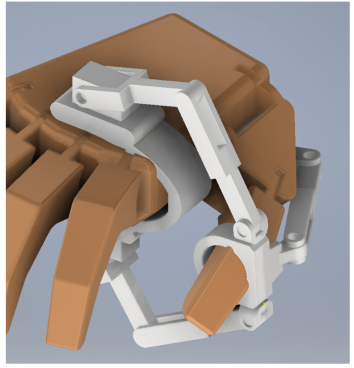

(c)

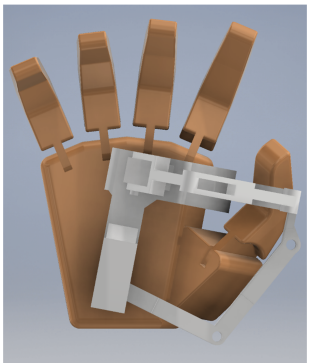

(d)

Figure 3. Conceptual design of proposed mechanism for the thumb rehabilitation: (a) 3D view, (b) overlapped with joint diagram, (c) Top view, (d) Bottom view (adapted by [18]).

In this paper, we discuss the geometric design, prototyping, and user test results taking into consideration the application of the mechanism for thumb rehabilitation therapy based on the methods of analysis from the previous works of [18-20]. The thumb trajectory was measured through a motion capture system to determine the target workspace and the required range of the thumb orientation angle. The motion capture system used is an optical type, which uses passive markers with an infrared light camera. Because this system does not necessarily install encoders with the actuators, the weight of the proposed mechanism can be reduced. Furthermore, it can measure the points located in the narrow space through the estimation of the center points between two markers. Collisions between the finger and mechanism may occur accidentally. This causes damage of the finger, and it affects the range of the workspace. For this reason, avoiding collision is considered in the design process. To avoid collisions and secure the workspace, the design considerations such as the placement of the actuator and the inclusion of link offset must be considered. 
For this reason, two candidates of mechanism design are considered in this paper. Each candidate has a different placement of the actuator. Because the workspace is affected by the actuator's placement, each workspace has a different range, even those set with the same design variables. To select a suitable candidate, two reachable workspaces are compared. The candidate, which has wider workspace than the other was chosen. The suitable link length of the prototype was determined through the coverage of the effective workspace in the target workspace. Considering these aspects, the prototype was manufactured and the user test was conducted considering AA and FE movements.

\section{2. (2-RRU)-URR Parallel Mechanism and Its Analysis Methods}

In the design process of this paper, the analysis methods that are reported from previous works $[18,19]$ are used. To help understand the contents of the paper, the mechanism explanation and the kinematic analysis are described briefly in this section.

The (2-RRU)-URR parallel mechanism is shown in Figure 4. In this Figure, chains ACE, BDF, and GHIJ are denoted as chains 1,2, and 3, respectively. The number of DOFs for this mechanism is zero, as calculated using Gruebler's formula. However, this mechanism actually exhibits three DOFs and this can be confirmed from the constraint wrenches[18]. Hence, this mechanism is categorized as a lower-mobility overconstrained mechanism. Furthermore, the proposed mechanism is composed of two planar chains, namely the planar motion generator (PMG: ACEFDB in Figure 4) and orientation motion generator (OG: OGHIJP). The PMG has formed the planar 6-bar linkage and The OG has formed the planar 4-bar linkage. for this reason, the kinematic analysis of each generator can be performed based on the planar kinematics [18,19]. As the mobility of this mechanism, the rotational mobility is along $x^{\prime}$-axis, the rotational mobility is along $z$-axis, and the translational mobility is along the $y$-axis. The mechanism achieves the target movement of $\mathrm{AA}$ and FE, by combining the three mobilities of the mechanism for the target movement as shown in Figure 5. In the case of AA, when the thumb is far away from the palm, and returns, its thumb position is controlled by the $y$ translational mobility. Simultaneously, the orientation of the thumb is changed, and this orientation is controlled by the rotational mobility along the $x^{\prime}$-axis. Regarding the FE movement, assuming that the orientation angle is not changed, the rotation mobility along the $x^{\prime}$-axis is set as constants. Furthermore, the thumb position is controlled in the $x y$ plane by combining motion from two mobilities of the rotational mobility along the $z$-axis and the translational mobility to the $y$-axis.

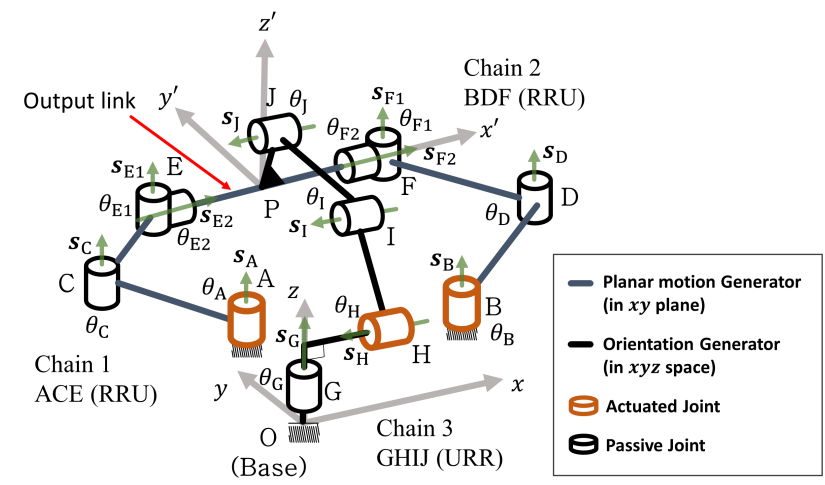

Figure 4. The diagrams of the (2-RRU)-URR parallel mechanism (adpated from [18]). 


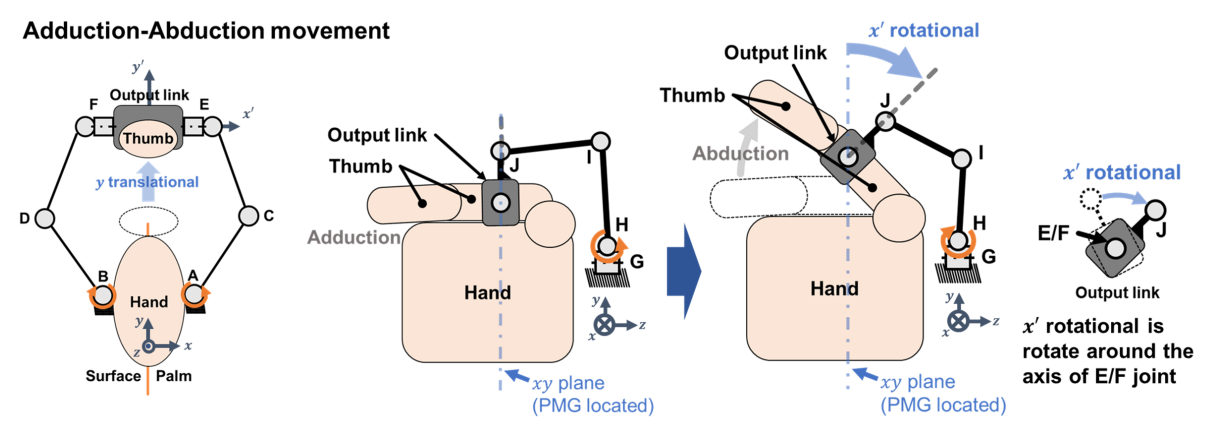

[PMG side]

[OG side]

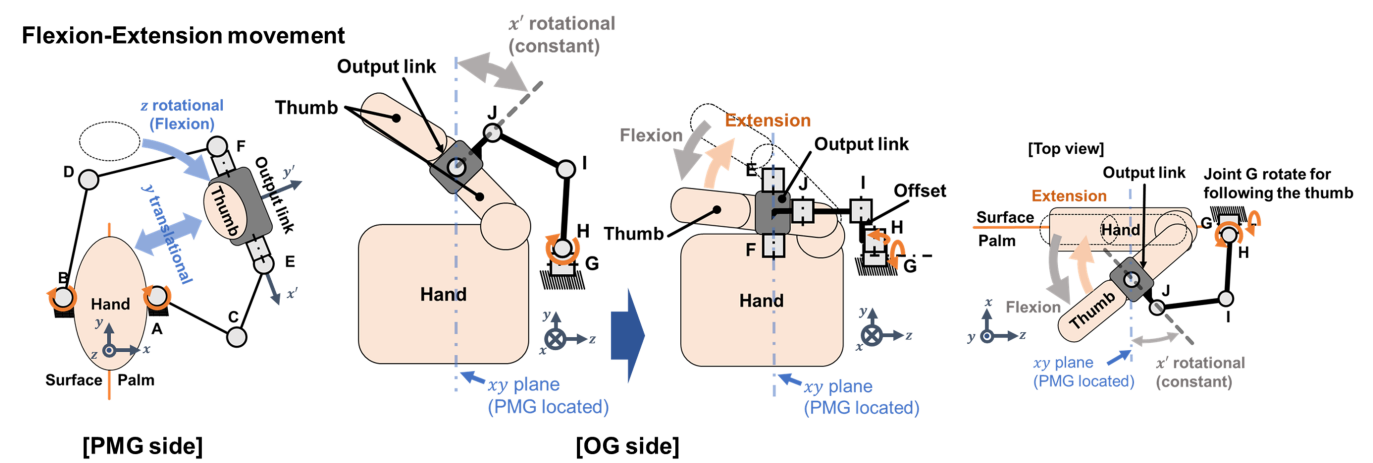

[OG side]

Figure 5. Explanation of the proposed mechanism's mobility in the cases of AA and FE movement. This figure explains the movements in the side of two parts such as PMG and OG.

Through these structural characteristics, the proposed mechanism can simply assist the thumb motions by controlling the attachment point like an end-effector type, and the complex motion of the CMC joint is not considered. However, because the attachment point is in the Proximal phalanx of the thumb finger, the motion of IP joints could not control. Moreover, the thumb finger's sliding motion may occur depending on the size of the hand due to the controlled motion provided in the $x y$ plane.

\section{Design Prototype for the Thumb Rehabilitation Therapy}

In our previous research, the mechanism's concept is theoretically considered, and it is focused on knowing the kinematic characteristics of the proposed mechanism. If the prototype designs without any considerations of the thumb rehabilitation requirements, the prototype is not practical, and it may not be attached well on the hand. Moreover, it cannot provide the thumb rehabilitation function as our expectation, and it may damage the thumb. Therefore, the investigations through prototypes which are designed based on the requirements in terms of functions, anatomy, kinesiology and practical implementations are very important to transform the proposed concept to the target users in order to prove the feasibility, performance and reliability of the proposed concept and even to envisage its future qualification. In this section, the design of the prototype with the consideration of the requirements is described. Based on the thumb measured data and the information of the kinesiology and the anatomy, the design requirement and the target of the movement were determined. To verify the performance of the proposed mechanism, experiments were performed to measure the trajectories of the thumb and its range of movement under two conditions of with and without attaching the prototype. The experiments involved the use of prototypes manufactured using a 3D printer. In this section, the experiment environment and system are described in terms of the specifications of each part, communication method, electrical/electronic specifications, and other aspects.

\subsection{Determination of the Target Workspace for Designing the Prototype}

Before manufacturing the prototype, the thumb measured trajectories of volunteers were measured. Certain data, such as the thumb trajectory, were measured using the motion 
capture system (OptiTrack Flex 13). Figure 6 shows the environment for the motion capture system. These measured data were used for various purposes, such as determining the target workspace and the source of the prototype's input trajectory. Because the proposed mechanism's position moves in the $x y$ plane by the PMG, the thumb measured data were projected into the $x y$ plane before using. The target workspace is the workspace in the $x y$ plane that includes all movement of the thumb measured data, and this is used as the design workspace. The input trajectory is the expected path of the output link and it is used as the input data when the proposed mechanism is controlled. Furthermore, this is also used to compare with the experimental data to prove the mechanism's performance and reliability.

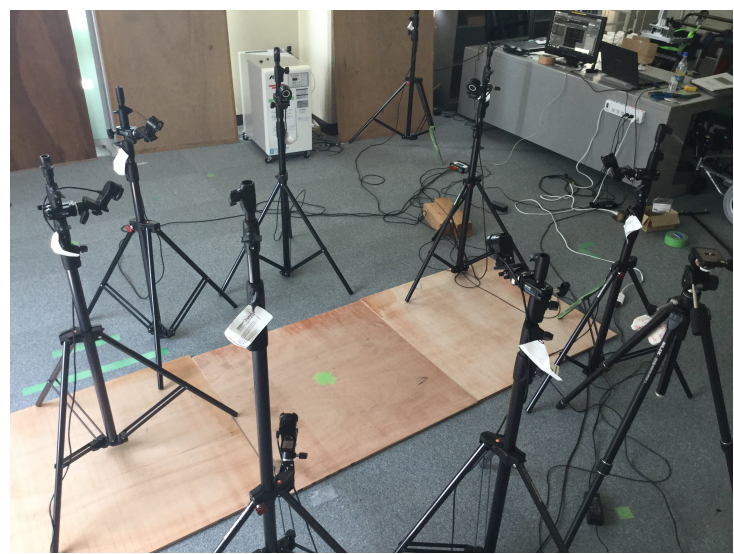

Figure 6. Measurement environment of motion capture system (adapted from [18]).

Because the camera used in the motion capture system detects the reflection of infrared rays, an error could easily occur due to light reflection. To reduce the reflection of infrared light from the skin surface, black gloves were used. To prevent the noise of the light, shades were used around the system to block external light. Figure 7 shows the markers on the hand for the measurement. As shown in Figure 7, seven markers were used. Markers 1 and 2 were the guide markers to indicate the hand direction. Marker 6 corresponds to the origin point, and marker 7 was used to set the $z$-axis as the line through the origin and marker 7 . Markers 3, 4, and 5 were used to measure the MP joint position and thumb tip. The thumb trajectory data was measured by using the motion capture system on twelve volunteers. The measurement safety was ensured in accordance with the experimental ethics of the university. The detailed information regarding this is presented in the experiment scenario section.
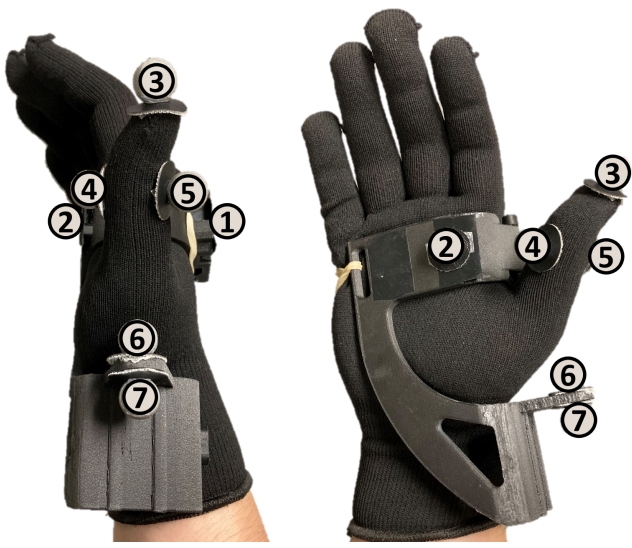

Figure 7. Markers for the thumb trajectory measurement.

When designing the proposed mechanism, its workspace must cover the two target movements: AA and FE. Due to the difference in the hand size of volunteers, the ranges of the movements are different. The thumb measured trajectories are three-dimensional 
data and are not located in a plane. Considering the structure of the proposed mechanism and its mobility, its position workspace is formed in the $x y$ plane. To determine the target workspace in this plane, the measured thumb trajectories are projected onto this plane. Here, the required workspace (= target workspace) is the workspace to cover all movements from the projected thumb trajectories. Figure 8 shows the AA and FE measured data of twelve volunteers in the $x y$ plane and the target workspace for PMG of the proposed mechanism. Each dot shown in this figure is the measured one for each movement. Those trajectories are classified by their shape. Therefore, the near rectilinear shape is the trajectory of the AA movement and the curved shape is the trajectory of the FE movement. In this figure, the total data consists of 24 sets of data $(12$ volunteers $\times 2$ required movements $=$ 24). To cover all movements of all volunteers, all measured data were plotted in the same space, and the boundary of the target workspace was determined by the one surrounded by the red line.

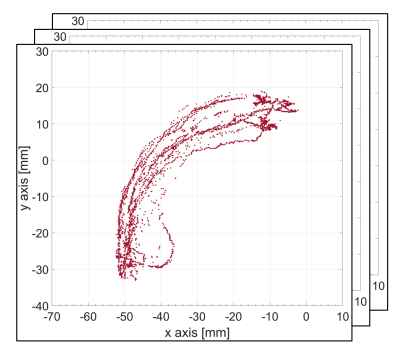

FE measuring data (12 EA)

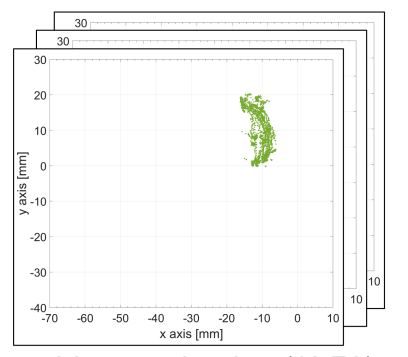

AA measuring data (12 EA)

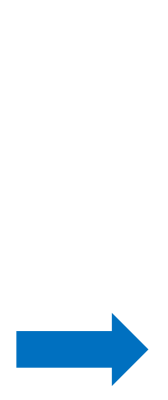

Overlapped all data

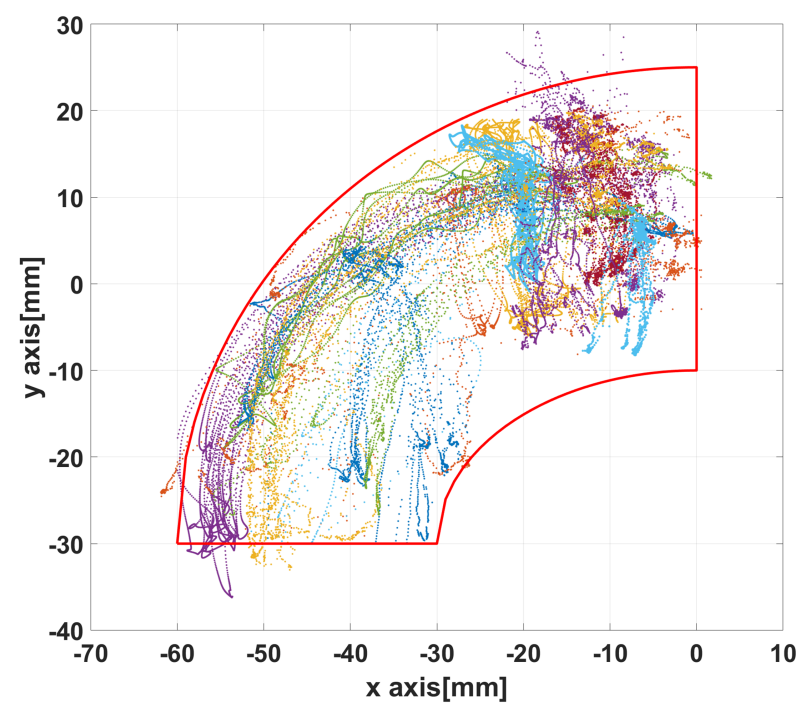

Figure 8. Target workspace defined by the measured trajectories of the thumb through motion capture system.

\subsection{Actuator Placement and Effect of the Workspace}

Because of the hand's narrow space, the actuator placement should be carefully determined in the geometric design. Of the the three actuators (each actuator adapted in joint A, B, and H), two actuators (A and B) are used for PMG and actuator H is used for OG. In detail, actuators A and B are installed on the palm and the hand's surface, respectively. In the $y z$ plane of the hand, the location of those actuators is similar or overlapped. Note that the axes of the passive joint $G$ and the active joint $H$ are crossed perpendicularly to each other similar to a universal joint. For this reason, when the placement of the third actuator is decided, the placement of the passive joint $G$ is also decided. Regarding the third actuator placement, if it is installed near the palm or the surface, the structure of the base part will be complex and there is the possibility that the collision between chains 3 and 1 or 3 and 2 occur. For this reason, the probable placement is chosen as near to the wrist. However, because the placement of the actuator affects the size of the reachable workspace, the placement is must still be considered in more detail. For the third actuator placement, two models were considered, as shown in Figure 9. Those candidates are designed with the third actuator placed near the wrist, but the detailed placements are different. 

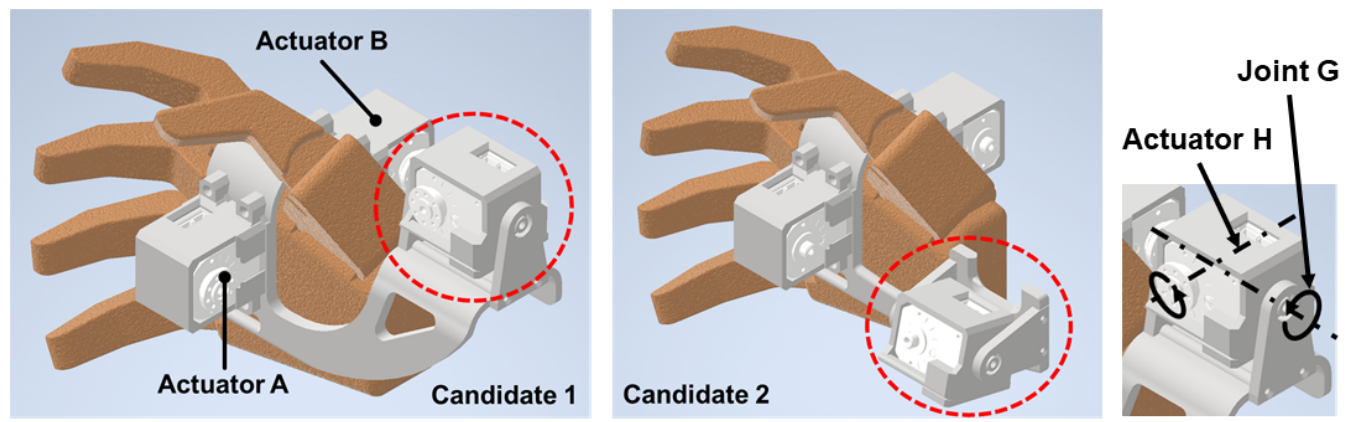

Figure 9. CAD images of two candidates, with the different placements of joints $\mathrm{G}$ and $\mathrm{H}$ : candidate 1 designed to be located behind the thumb, and the candidate 2 designed to be located beside the wrist.

In the first candidate, the third actuator is placed on the side of the wrist. In the coordinate system in the figure, the third actuator's position of the $x y$ plane matches the origin point. In the second candidate, the placement of the third actuator is designed to be nearby the wrist. Based on the above-mentioned coordinate system, its actuator position of the $x y$ plane is not in the origin point. Regarding the position of the third actuator along the $z$ axis, both design candidates are set to the same value of zero. Furthermore, an offset was included to reduce the collision of the hands and ensure the parallelization of the third chain. Figure 10 illustrates two candidates and its difference.
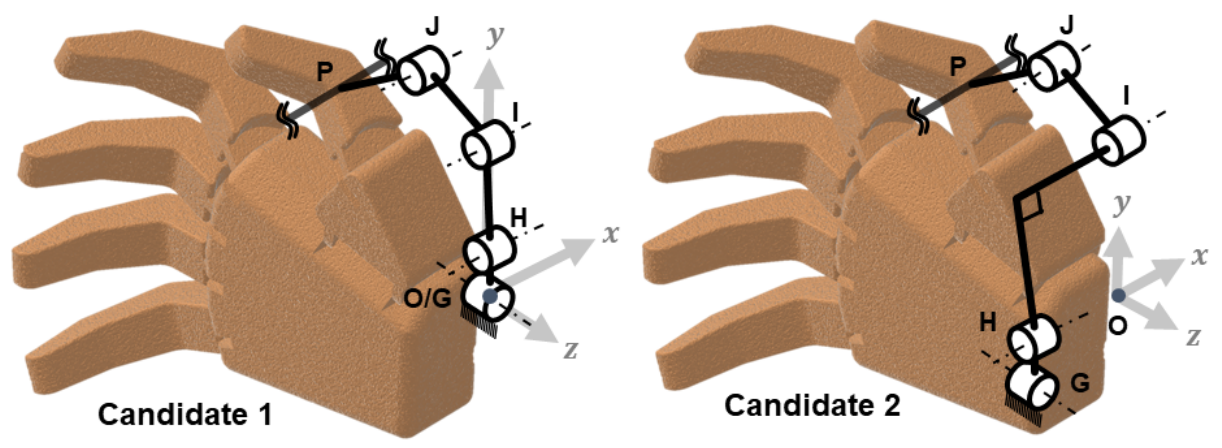

Figure 10. Difference of each candidate and configuration of the third chain for each candidate. (The actual distance between joint $\mathrm{H}$ and $\mathrm{G}$ is zero).

To find a suitable design, a workspace comparison of the two candidates is carried out. Table 1 shows the relevant parameters. On the basis of PMG, $l_{\mathrm{AC}}, l_{\mathrm{CE}}, l_{\mathrm{BD}}$, and $l_{\mathrm{DF}}$ were set to have the same link length. Furthermore, $l_{\mathrm{HI}}$ and $l_{\mathrm{IJ}}$ were set to have the same link length based on OG. The reason for those settings is for the simplification of the calculation for the design. Therefore, the design parameters of links are set as $l_{1}\left(=l_{\mathrm{AC}}, l_{\mathrm{CE}}\right.$, $l_{\mathrm{BD}}$, and $\left.l_{\mathrm{DF}}\right)$ and $l_{2}\left(=l_{\mathrm{HI}}\right.$ and $\left.l_{\mathrm{IJ}}\right)$. The parameters, such as $l_{1}, l_{2}$, and joint positions for two candidates, were the same in both cases. Other parameters such as offset, $l_{\mathrm{EF}}$, and $l_{\mathrm{JP}}$ are set as constant values with respect to the size of the hand. Moreover, the range of workspace of the output link position was given as random values. Based on these parameters, the reachable workspace of each candidate was derived. Figure 11 shows both reachable workspaces. The reachable workspace for the first candidate exhibits a vertical, tower-like workspace. On the other hand, the second candidate's workspace exhibits a large width in the direction of the $x$-axis while having the same height as the first candidate. Moreover, each workspace as is compared to the target workspace (red marked area in Figure 8), as shown in Figure 12. In terms of the target workspace, the second candidate covers more of the target workspace than the first and it also completely covers the target workspace. This phenomenon is related to the perpendicular connected structure of chain 3 and the output link. The workspace is mainly drawn through this structure as a circular-like shape, 
and the constraint of chain 1 and chain 2 cause the change in its shape. In the result of Figure 12, the distance between the joint $G$ and actuator A or B caused an increasing constraint of chains 1 and 2. In other words, when the distance increased, the area of the reachable workspace decreased in the $x$-axis direction. Therefore, the second candidate appears to be a reasonable choice for designing the prototype to be attached to the users, even though the offset makes the structure relatively complex.

Table 1. The parameters of two candidates which have different base design.

\begin{tabular}{|c|c|c|c|c|c|c|}
\hline \multirow{2}{*}{$\begin{array}{c}\text { Parameter } \\
\text { Joint }\end{array}$} & \multicolumn{2}{|c|}{ Both } & \multicolumn{2}{|c|}{ Candidate A } & \multicolumn{2}{|c|}{ Candidate B } \\
\hline & A & {$[-49,-52,-69]$} & G & {$[0,0,0]$} & G & {$[-50,-55,0]$} \\
\hline Position & B & {$[47.5,-52,-69]$} & - & - & - & - \\
\hline$[x, y, z](\mathrm{mm})$ & $\mathrm{O}$ & {$[0,0,0]$} & - & - & - & - \\
\hline \multirow{4}{*}{ Link Length (mm) } & $l_{1}$ & 64 & Offset & 0 & Offset & 20 \\
\hline & $l_{2}$ & 50 & - & - & - & - \\
\hline & $l_{\mathrm{EF}}$ & 58 & - & - & - & - \\
\hline & $l_{\mathrm{JP}}$ & 24 & - & - & - & - \\
\hline \multirow{2}{*}{$\begin{array}{c}\text { Given position of } \\
\text { output link point }(\mathrm{mm})\end{array}$} & $\mathrm{x}$ & $\begin{array}{l}-180 \leq x \leq 180 \\
\text { (Interval: } 1 \mathrm{~mm})\end{array}$ & - & - & - & - \\
\hline & $\mathrm{y}$ & $\begin{array}{l}-40 \leq y \leq 180 \\
\text { (Interval: } 1 \mathrm{~mm} \text { ) }\end{array}$ & - & - & - & - \\
\hline
\end{tabular}

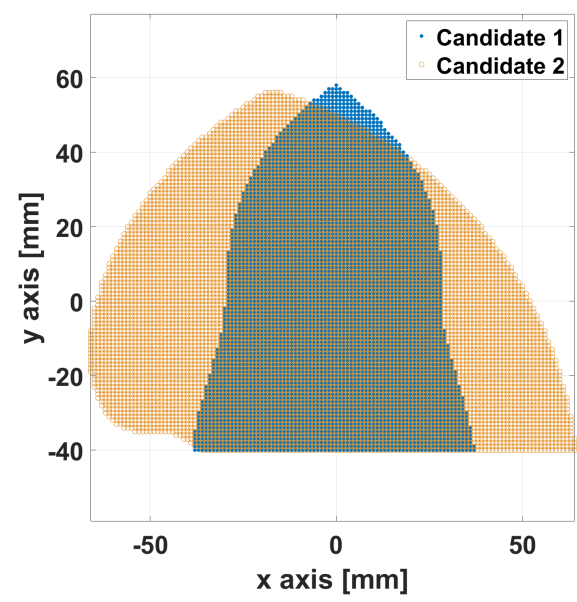

Figure 11. The derived reachable workspace for each candidate.

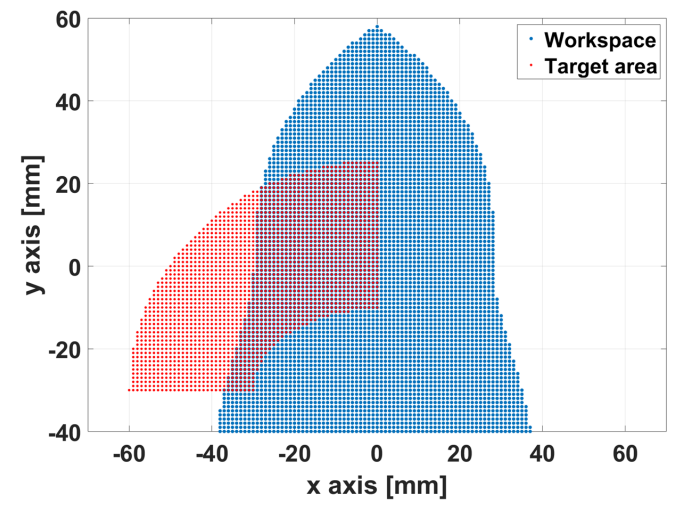

(a) Candidate 1

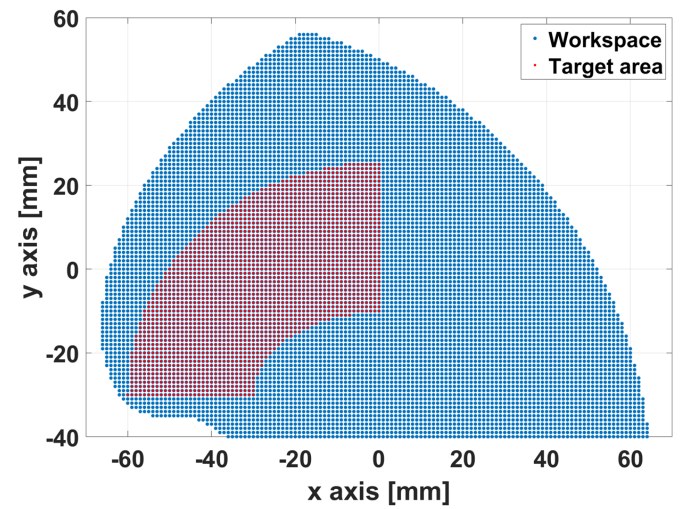

(b) Candidate 2

Figure 12. The workspace when the target workspace overlapped in same coordinate space: (a) Candidate 1, (b) Candidate 2. 


\subsection{Prototype}

Considering the aforementioned results, a prototype of the proposed mechanism was developed. Figure 13 shows the CAD drawing of the prototype. To adapt to different hand sizes, the base module was designed to incorporate three parts. The three parts are called the upper base part 1, upper base part 2, and under base part, respectively, and each part is connected by a passive revolute joint. One actuator was installed in the upper base part 1 and the other actuators were installed in the under base part. The upper base part 2 was passively attached to the hand surface. Through this structure, the base module can wrap the hand without needing to consider differing hand thicknesses. When the mechanism is placed on the hand, the parts were fixed using a Velcro strap, as shown in Figure 14. In terms of hand thickness, the mechanism was designed to fit hands with a thickness of more than $33 \mathrm{~mm}$, in consideration of the human measurement data. Thus, the base module can adapt be fixed to hand sizes with a high thickness. Moreover, the shape of each part is complex to provide an ergonomic shape. To fabricate the complex shapes, most parts of the prototype were fabricated using a 3D printer (Markforged Mark two), and the parts were reinforced with carbon fiber. Furthermore, the third actuator, connected to chain 3, was placed near the wrist and thumb CMC joint. This placement allows the center of weight of the mechanism to be close to the center of the hand. The manufactured prototype and the attachment state are shown in Figure 15. The weight of the prototype was $317 \mathrm{~g}$ (including the actuators). Figure 16 shows the parameters of the prototype's link lengths.

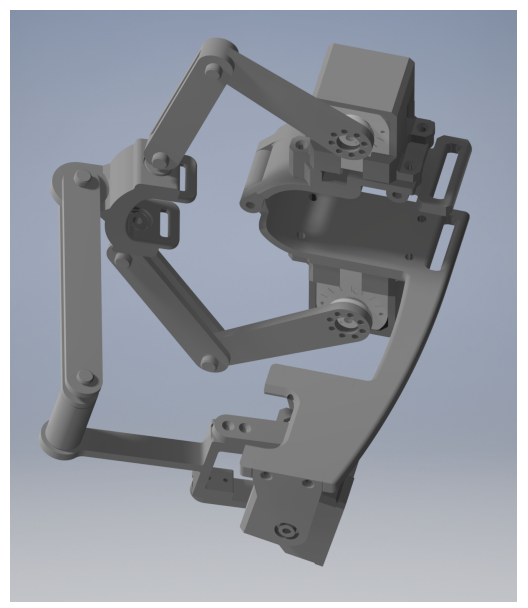

Figure 13. CAD drawing of the proposed mechanism.
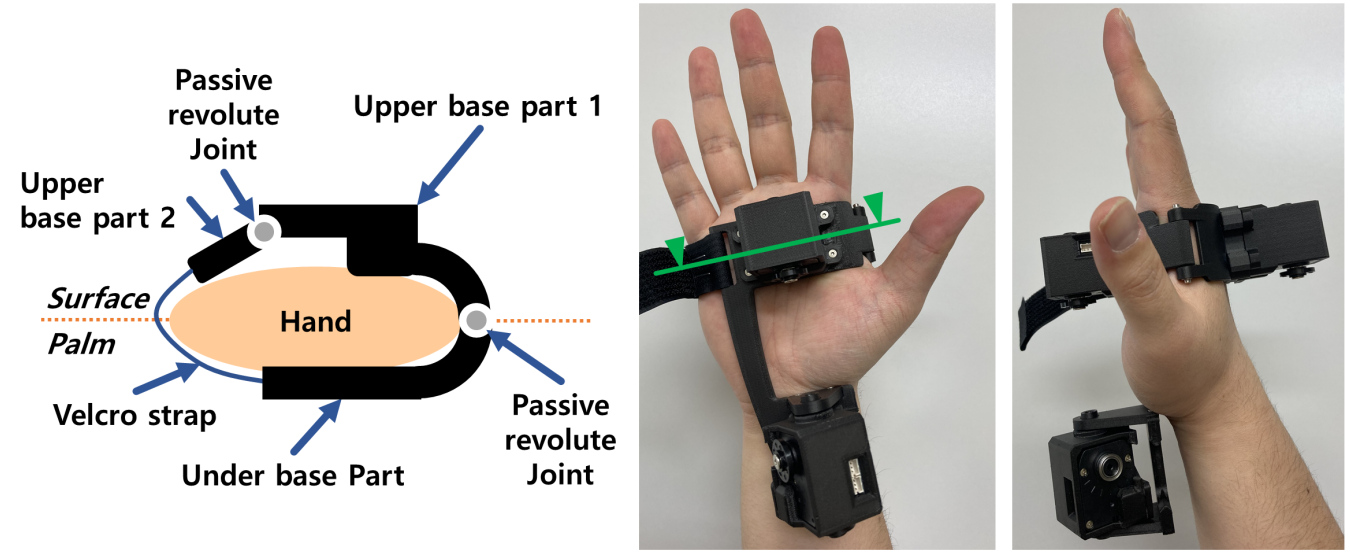

Figure 14. The cross-section of base parts and the attached state of the base with hand. 

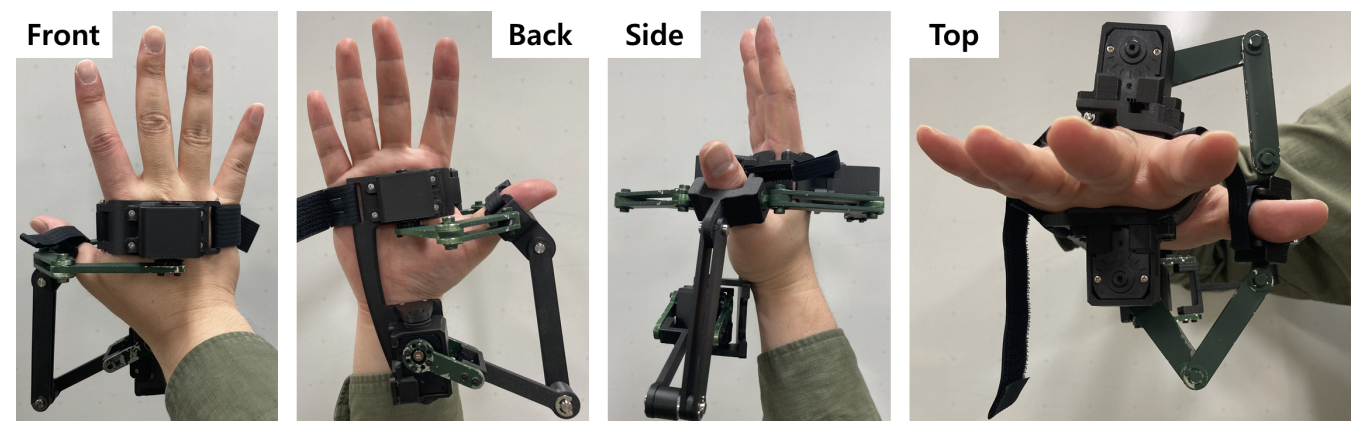

Figure 15. Manufactured final design of prototype.

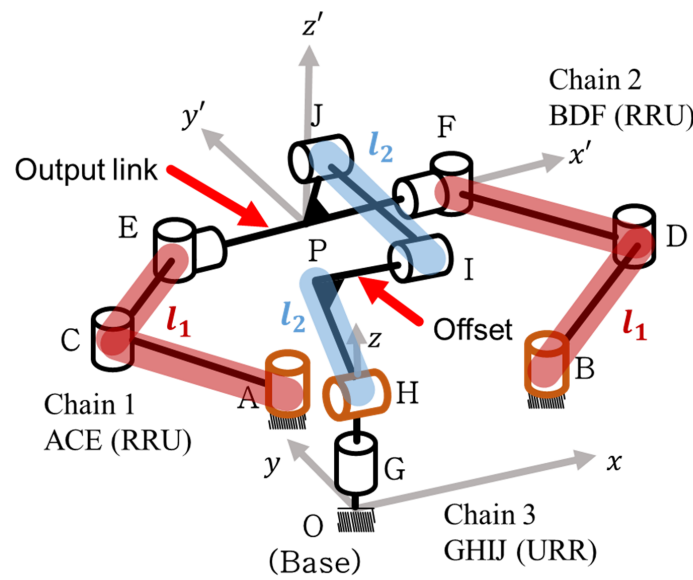

\begin{tabular}{c|c}
\hline Link & length $(\mathrm{mm})$ \\
\hline$l_{1}\left(l_{\mathrm{AC}}, l_{\mathrm{BD}}, l_{\mathrm{CE}}, l_{\mathrm{DF}}\right)$ & 56 \\
\hline$l_{2}\left(l_{\mathrm{HI}}, l_{\mathrm{JI}}\right)$ & 87 \\
\hline$l_{\mathrm{EF}}$ & 58 \\
\hline$l_{\mathrm{JP}}$ & 24 \\
\hline Offset & 20 \\
\hline
\end{tabular}

Figure 16. The design variables of each link for prototype.

\subsection{Experiment System and Control}

The experimental system consisted of three elements, specifically, the proposed mechanism, driving/control part, and control algorithm. The driving/control part contained all the electrical parts needed to control the mechanism. The algorithm was the embedded in a program provided to the processor. The driving/control part consisted of the micro-controller unit (MCU), servomotor, and signal conversion board. The servomotor (KRS-3304R2 from Kondo Corporation) is a DC type motor, controlled through UART communication, with the electric power being $6.0 \mathrm{~V} / 2.0 \mathrm{~A}$. Table 2 presents the detailed actuator specifications.

Table 2. Specification of servo motor used in experiments.

\begin{tabular}{cc}
\hline Operating power & $6.0 \mathrm{~V} \mathrm{2.0} \mathrm{A}$ \\
\hline Maximum Torque & $1.10 \mathrm{~N} \cdot \mathrm{m}$ \\
\hline Maximum Speed & $0.11 \mathrm{~s} / 60^{\circ}$ \\
\hline Size & $32.5 \times 26 \times 26 \mathrm{~mm}^{3}$ \\
\hline Weight & $33.7 \mathrm{~g}$ \\
\hline Maximum operating angle & $\pm 135^{\circ}$ \\
\hline
\end{tabular}

Because the actuator uses only one signal line for the transmitting and receiving information, circuits to realize communication between the MCU to the servomotor were established. The signal conversion board converted the two signals into one signal for the servomotor and divided the signal into two signals for the MCU. The control processor used was an Arduino UNO based on a $5.0 \mathrm{~V}$ logic. Figure 17 shows the overall connection and communication status of the driving/control parts. Position control was implemented for control of the actuators. 


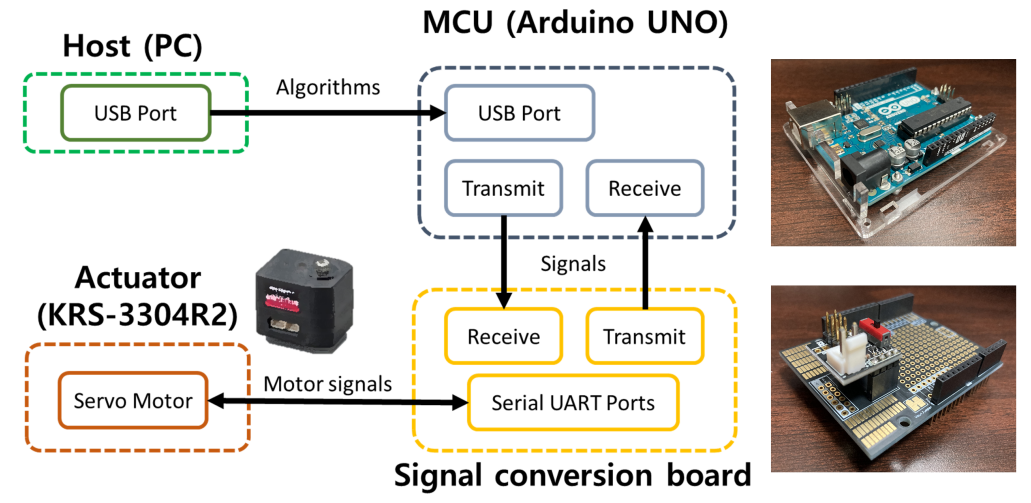

Figure 17. Diagram maps of the experimental system.

\subsection{The Planned Trajectory and the Range of the Orientation Angle}

To determine the input trajectory for the prototype, trajectory planning was implemented using the measured thumb trajectory. The planned trajectory was calculated using the center point of the breadth of the raw thumb trajectory. In the case of AA, its form is similar to a long rectangle and the pattern of the trajectory moves along the $y$ axis. Therefore, the planned trajectory is easily calculated from the average of the $x$ axis value in regard to each $y$ value. Because the form of the FE is curved, the calculation is separated into two parts. The upper part of the FE trajectory has a wide range along the $x$ axis. The lower part has a small range along the $x$ axis and its trajectory is formed along the $y$ axis. For this reason, the calculation of the lower part trajectory is the same as the calculation done for AA. For the upper part, the calculation is done using the average of the $y$ value. Figure 18 shows the raw measured data and calculation result of the trajectory planning. From this figure, the dots in the planned trajectory (referred to as steps) are input points for the inverse kinematics for the required position of P. In addition, the points passed on the planned trajectory were selected. Due to the FE trajectory being longer than AA, eight and three dots for the FE and AA movement were selected, respectively. In controlling the prototype, the output link passes through dots one by one, and a time delay is given between the dots to control the trajectory speed. Using the selected dots, the required angles of the actuation joints A and B were derived through inverse kinematic analysis. It was confirmed that the planned trajectory was located within the target workspace.

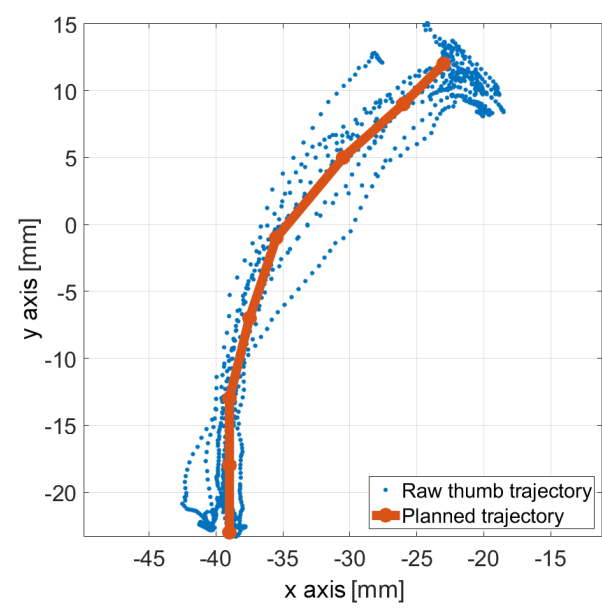

Figure 18. An example of the trajectory planning result of FE movement.

In addition, the range of the orientation angle of the thumb was decided. Comparing the movements of $\mathrm{AA}$ and $\mathrm{FE}, \mathrm{AA}$ has a larger range of thumb orientation angle compared to the FE movement. For this reason, the range of the orientation angle is determined from the range of the AA movement. In the literature from [21,22], the total range of the AA movement was reported as $40^{\circ}$. Considering the situation wherein the mechanism is 
attached to the hand as shown in Figure 19, the minimum orientation angle is set as zero. While the thumb can move more towards the palm, its movement is blocked by the base part. For this reason, the range of the orientation angle was set as $0^{\circ}$ to $40^{\circ}$ for the experiment. In the case of AA, the thumb is located in the palm of the hand and the orientation angle along the $y$ axis $(\Psi)$ is zero for the adduction movement. Furthermore, when the thumb moves far from the palm (abduction), $\Psi$ increases. Therefore, the orientation angles for the three steps of AA movement were set as $0,20,40^{\circ}$ from adduction to abduction movement. Regarding the FE movement, a constant angle of $40^{\circ}$ was given to all steps of the FE planned trajectory. This orientation angle was used to derive the required angle for the actuation angle $\mathrm{H}$ through inverse kinematics.
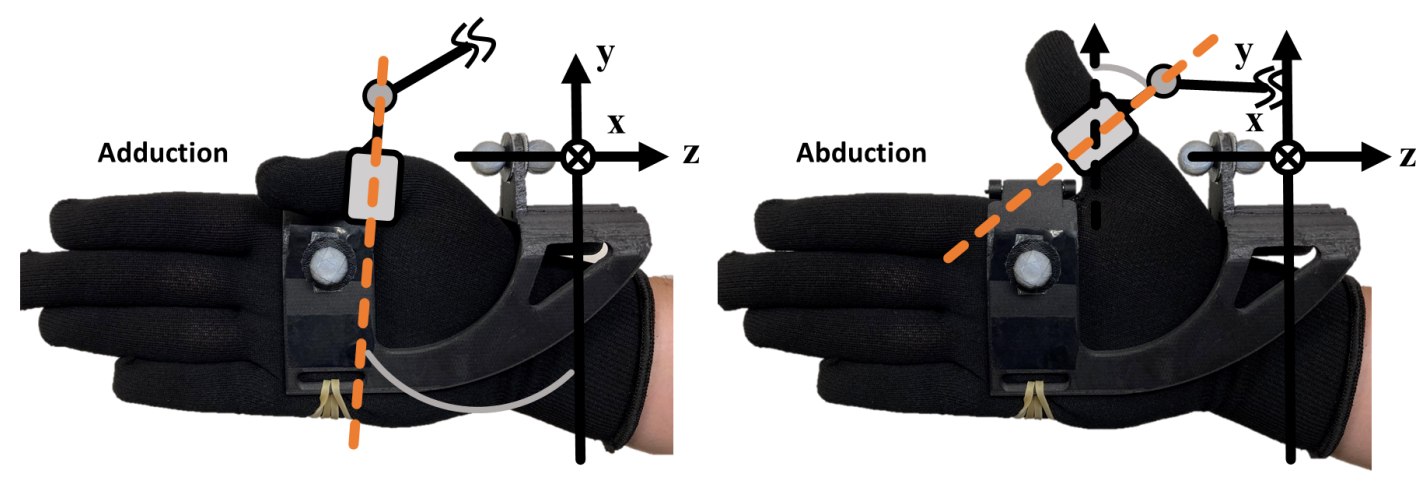

Figure 19. Range of the required orientation angle when thumb equipped the output link (Adduction-Abduction movement).

\section{User Experiments}

Because the proposed mechanism is to be applied for rehabilitation therapy, it was necessary to verify the presented movement while considering the effect on the human hand. In the experiment, each volunteer's planned trajectory and the range of the required orientation angle were used to control the prototype. When the output movement of the prototype is following through the input trajectory, we assume that the prototype has the feasibility of the thumb rehabilitation robot. For this reason, the control of the prototype for two movements were done and the position of the output link was measured. After measuring, the experimental data with the input trajectory was compared. Moreover, an experiment was conducted on publicly recruited volunteers to perform a performance evaluation and identify any unexpected problems by obtaining feedback through a questionnaire.

In the experiment, the two movements, namely AA and FE, were considered. To control the prototype, the thumb trajectory, which was measured with the motion capture system in Figure 6, was used. The thumb data were measured before the experiment by using the prototype and these were also used in the determination of the target workspace of Figure 8. The volunteers were briefed prior to the experiment regarding the thumb measurement. Moreover, these data were considered to be the target group and the measured thumb data were used for generating the planned path of the experiment. The planned path was an individual path for each user and did not use the same path for other users. Before the experiment, the user was asked to sit near the camera of the motion capture system, and the prototype was attached to his/her right hand. The movement was provided by the prototype to the user five times, for each movement. Figure 20 shows the experimental scenario. Because the results of the experiment were not expected to be influenced by factors such as the temperature and humidity of the room, these factors were not limited. To measure the trajectory of the prototype performance, motion markers were attached to the prototype during the experiment. Figure 21 shows the attached markers on the prototype. The measurement point is the middle point of four markers: 8, 9, 10, and 11 . In the experiment, the following safety clauses were implemented: 
- The torque of the used actuator was less than that of the thumb.

- To prevent damage to the human body, mechanical and electrical safety measures were implemented, such as the installation of electrical circuits to prevent overload and electrical back-flow.

- Two emergency stop switches were used. The first emergency stop switch was provided to the users to stop operation during the experiment if they felt any pain or discomfort. The second switch was installed for the organizer of the experiment to stop the operation of the device.

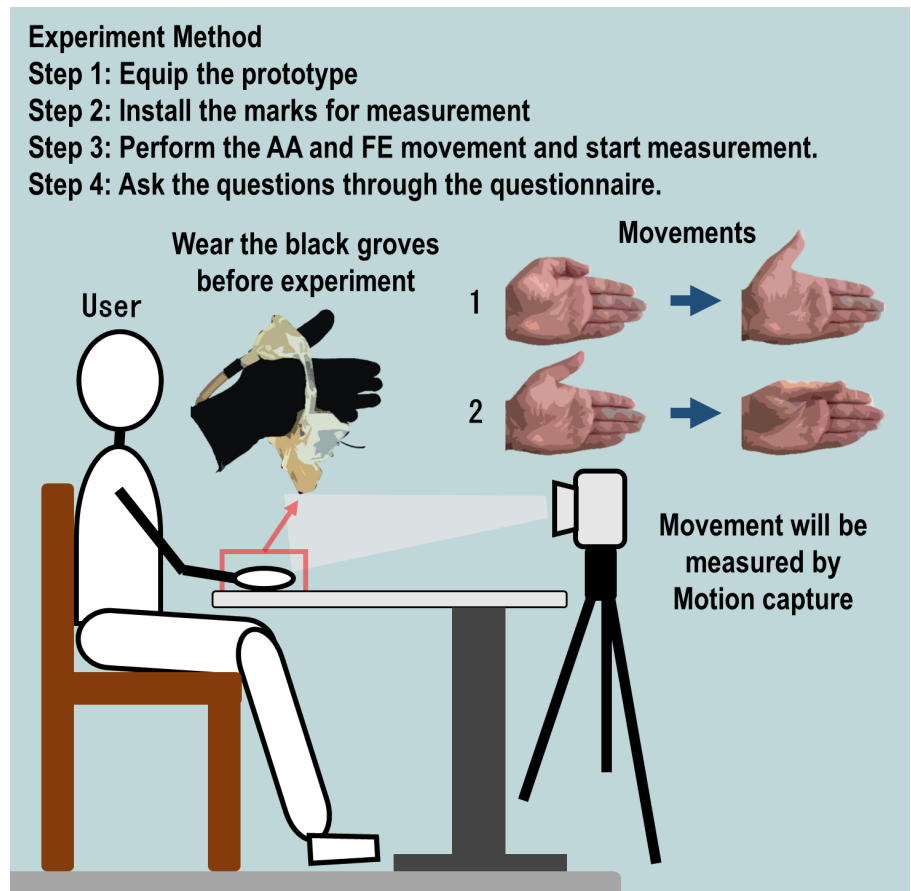

Figure 20. Concept of the experimental scenario.

The experiment scenario and safety clauses were approved by the research ethics committee of Tokyo Institute of Technology (Registration number: A19190).

Figure 22 shows the experiment results pertaining to the FE movement of four users which was controlled using the prototype. These experiment data were measured as three-dimensional data using the motion capture system. Because the reachable workspace was located in the $x y$ plane, the figures are shown in the $x y$ plane. The blue dot and line represent the planned trajectory, which are generated from the thumb trajectory while the users did not have the prototype attached, and the orange dots correspond to the measurement data of the thumb trajectory provided by the prototype. In the figure, the orange dots indicate the difference from the planned trajectory (marked by the blue dots). From the result of Figure 22, the position of the output link shows that the prototype generates a trajectory that is mostly similar to the planned trajectories. Thus, the prototype is capable of controlling the FE movement.

To confirm the FE movement in detail, the orientation angles around the $z$ axis in the $x y$ plane of thumb and output link were compared (this angle is not $\Psi$ ). Figure 23a shows the explanation of the two angles, and Figure 23b shows the two angles: the blue line is the thumb orientation angle, and orange is the output link orientation angle. Those two data were not simultaneously measured, but the patterns of the movements and the trajectories are largely the same. From the result of Figure 23, we know that relative motion, which is the sliding motion between hand tissue and bone, between the two angles exists. Therefore, we estimate that the relative motions occur between the output link and the base part. Because of the effects of the relative motion, the tip of the trajectory of the experiment is not expected to match well with the planned trajectory in the practical experiment shown in 
Figure 22. To improve the accuracy of the generated trajectory, the relative motion should be reduced, which will be done in future work.

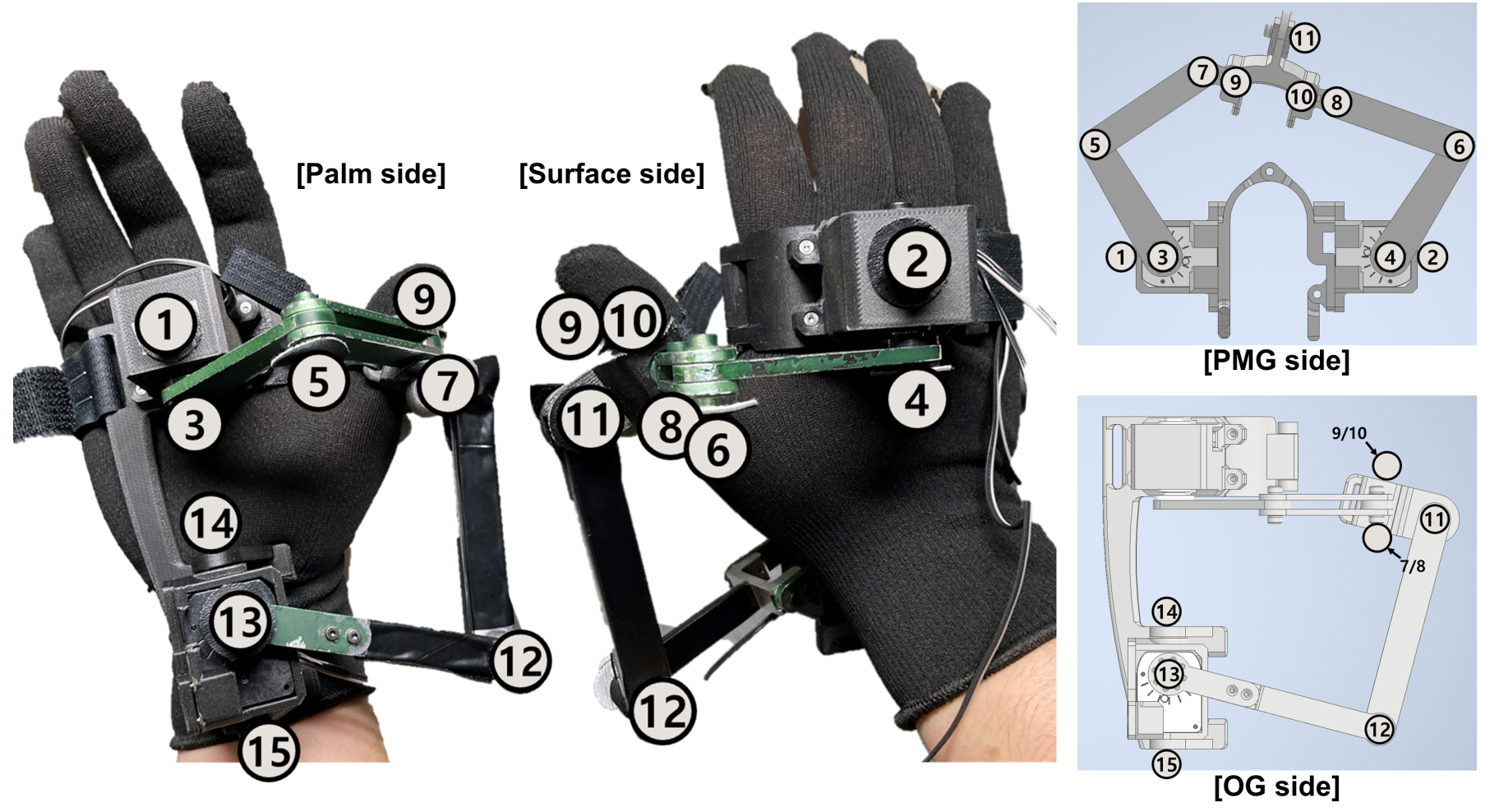

Figure 21. Measurement markers on the prototype in the experiment.

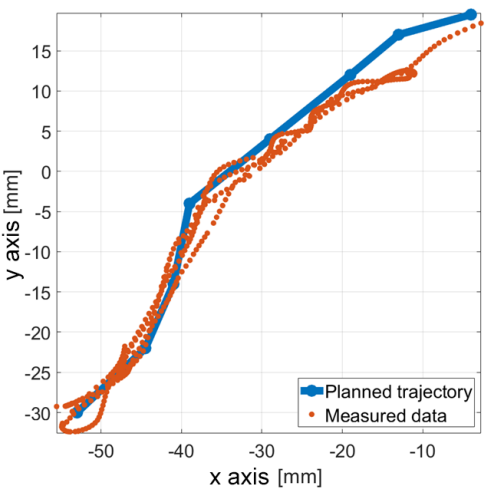

(a)

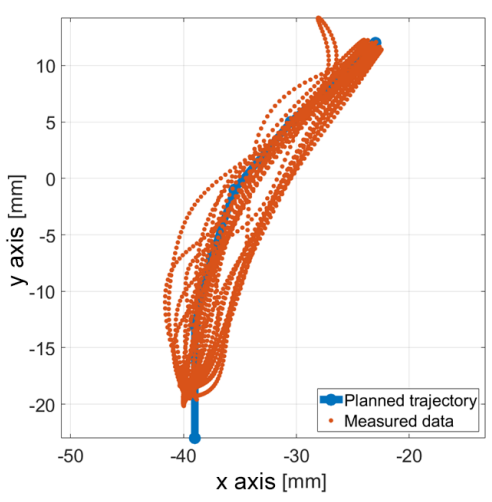

(c)

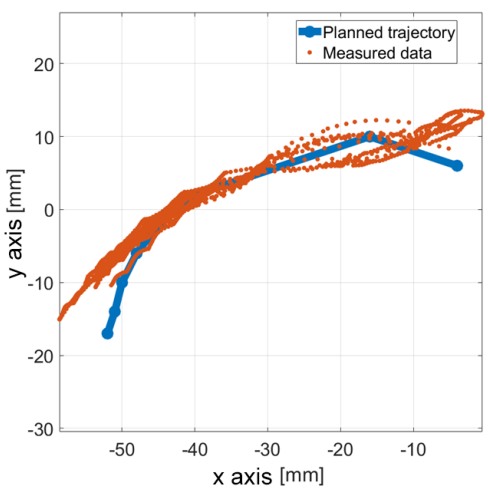

(b)

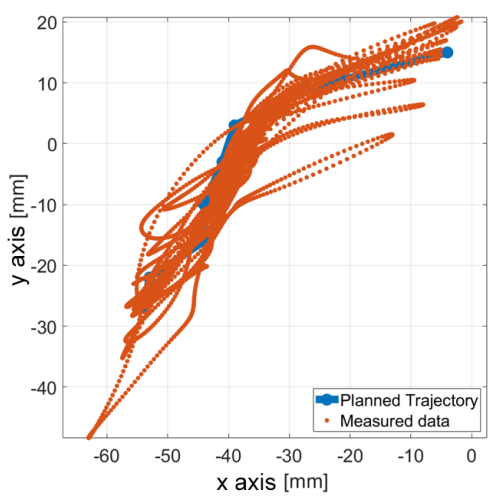

(d)

Figure 22. Comparison the planning trajectory and measurement data for each person in the FE movement. 

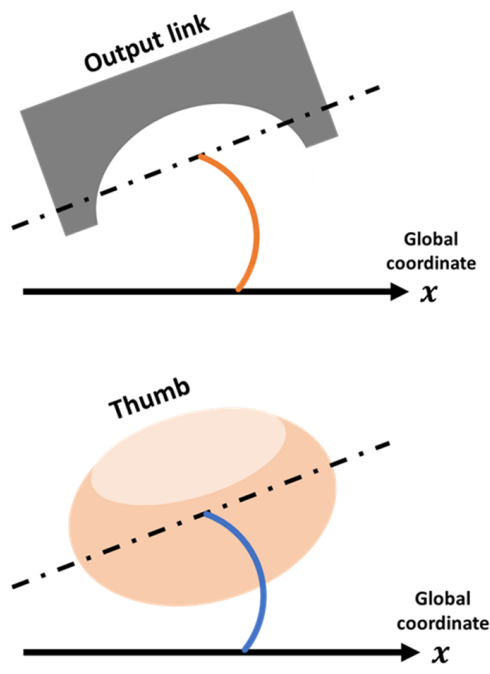

(a) Explanation of orientation angle

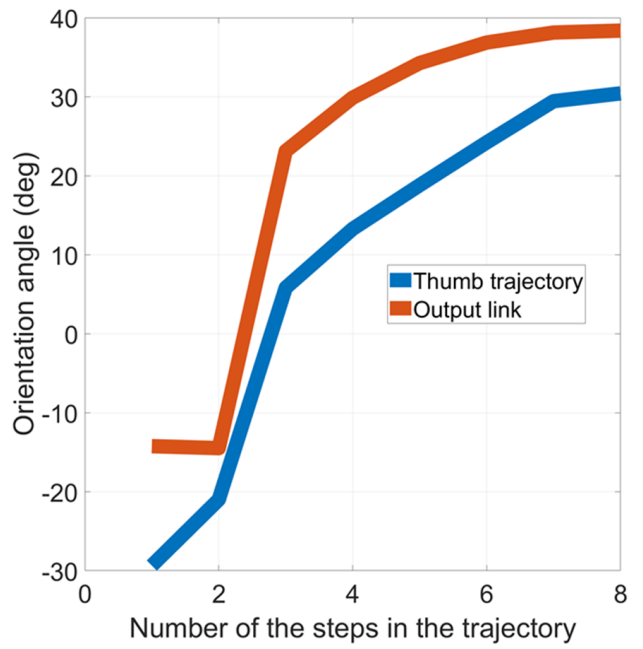

(b) Two orientation angles

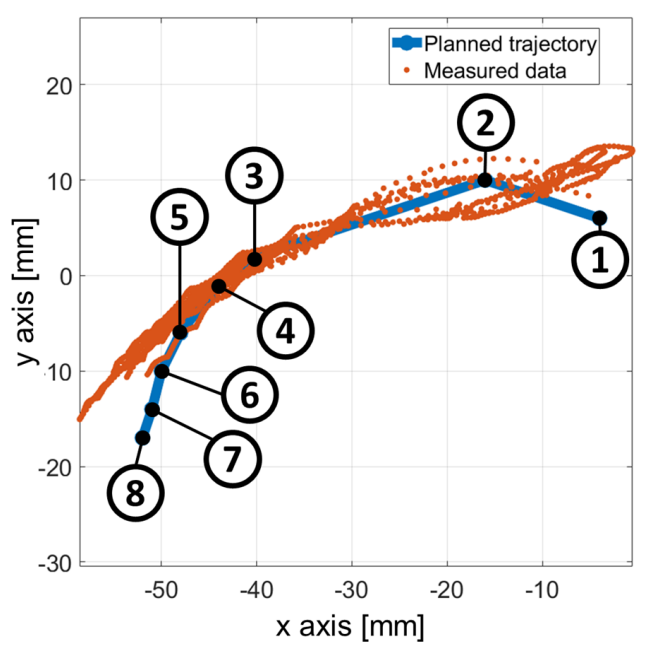

(c) Steps in the planned trajectory

Figure 23. The orientation angles (around $z$ axis in the $x y$ plane) of the thumb measured data, and the output link of the experimental data and the explanation of the steps on the used planned trajectory: (a) explanation of orientation angle, (b) two orientation angles, (c) steps in the planned trajectory.

Figure 24 shows the results for the AA movement. The measured data are significantly smaller compared to the planned trajectory data. From this result, it is expected that the motion transmission from the actuator was not well done or the actuator did not work well. This means that the prototype moved in a small range and its behavior included vibratory motion. Figure 25 shows the simulation results for the planned trajectories of the above-mentioned example. The different sub-figures show the configuration of the mechanism when $P$ is assigned with respect to the planned trajectory shown in Figure 24. It can be noted that actuation joint A does not change considerably from (a) to (c). This experimental result is expected due to the lack of torque.

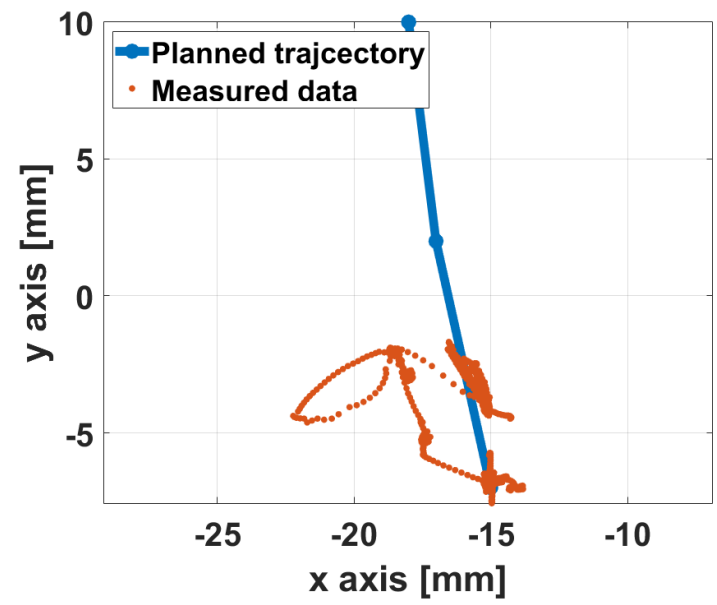

Figure 24. Comparison the planning trajectory and measurement data in the AA movement. 

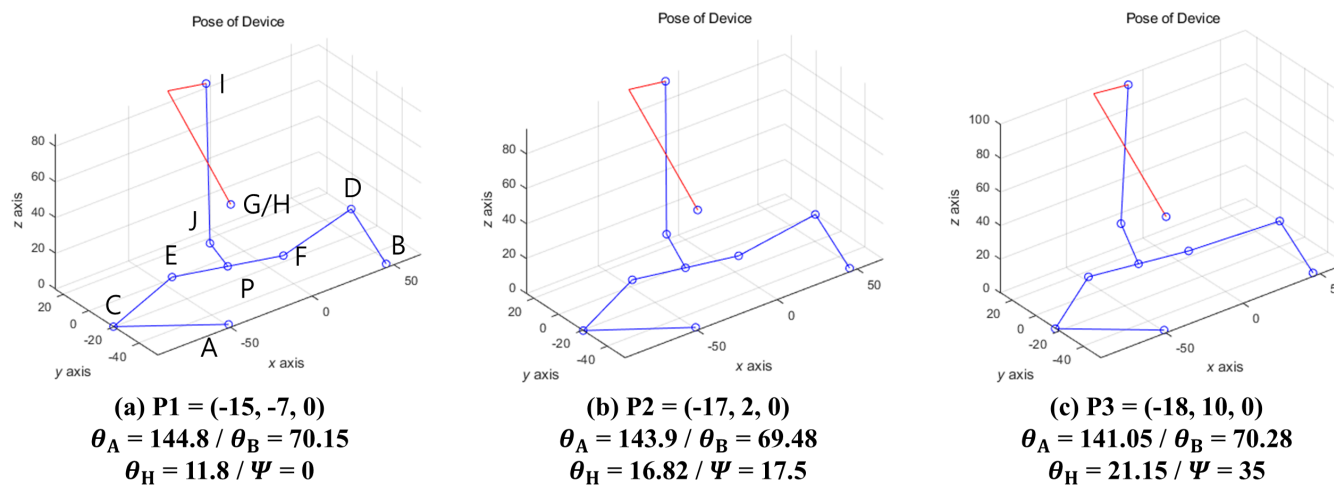

Figure 25. The simulation results of the planned trajectories of the above-mentioned experiment.

Because of the lack of actuator torque in the experiment of AA movement, a manual movement, which provides assistance to the actuators by hand without electric power, was carried out. This was a simple test done to confirm the prototype performance when the actuators have enough torque. When AA movement was performed, the result, the target workspace, and the planned trajectory are shown in Figure 26. The green dots indicate the output link position of the measured data wherein the prototype was moved by assisting the actuator by hand. Because this data was obtained while moving the actuator by hand, it does not perfectly follow the planned trajectory. From this result, it can be seen that the measured data of the manual movement has a larger trajectory, and it protrudes from the range of the target workspace. Therefore, if the actuator is capable of enough torque output, the prototype is expected to be capable of controlling AA movement. As for future work, additional analysis of the required torque while considering the weight of the prototype for application of the thumb rehabilitation will be carried out.

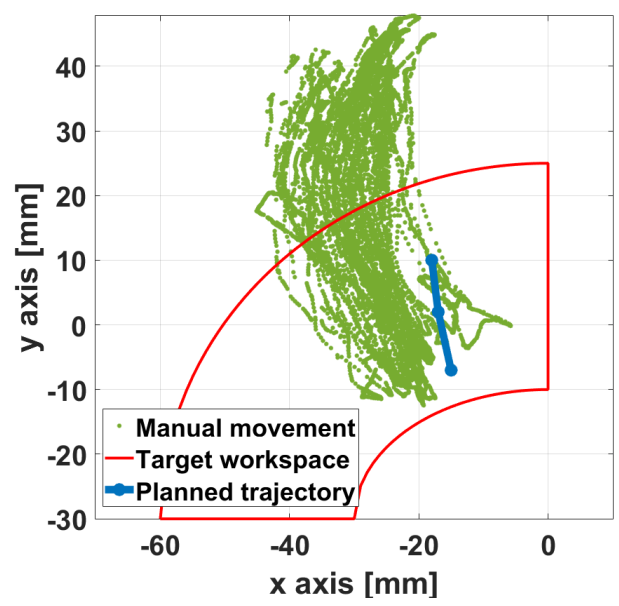

Figure 26. The measurement data of the AA movement in the case of the attached prototype moving by hand.

\section{Discussion and Conclusions}

In this paper, the geometrical design, prototyping, and user test of the proposed mechanism were described to be adapted for application in thumb rehabilitation therapy. The proposed mechanism is a hybrid type, which combines the exoskeleton and the endeffector type mechanisms. The structural characteristics allow the thumb CMC joint's complexity to not be considered in the mechanism design. The design requirements were determined such that the thumb rehabilitation's proposed mechanism can be adapted to the anatomical and kinesiological information. The thumb trajectory was measured using an optical motion capture system to determine the target workspace. Two design candidates, which had different actuator placements, were considered in order to avoid collisions between the mechanism and hand. The sizes of the three workspaces (target workspace, the 
workspace of candidate 1 , and the workspace of candidate 2) were compared while keeping the two candidates' design variables the same. Because candidate 2 was found to have a wider workspace than candidate 1 while being able to cover the target workspace, candidate 2 was selected as a suitable design. Based on these considerations, a prototype was manufactured. To prove the suitability and performance of the mechanism for application in thumb rehabilitation, user tests were carried out. User testing was performed using the prototype on volunteers. The input data were determined from the results of the trajectory planning method. Two target movements were provided: FE and AA.

The user experiment with the prototype was carried out to verify the effectiveness of the prototype for the thumb rehabilitation therapy. In the experiment, two target movements were provided: FE and AA. The results for the FE movement were largely satisfactory following the planned trajectory. Because the tip of trajectories did not match with the input trajectory well, the relative motion between the thumb and output link was compared. The results showed that a relative motion exists in both ends of the trajectory and it causes a small effect on the control accuracy of the prototype. On the other hand, the AA movement cases were not satisfactory. To investigate these, a manual moving test of the prototype using the AA movement was done by assisting the actuators by hand. Through this test, it was shown that the range of the output trajectory was longer than the planned one, and it could cover the target workspace. Thus, the prototype has the potential to perform the AA movement. To sum up these findings, the prototype has the possibility to adapt for thumb rehabilitation though the prototype used in this paper has some limitations. To improve the proposed mechanism for thumb rehabilitation, actuation selection will be necessary by performing static analysis on the prototype. Future work on the mechanism design while considering the design factors calculated from the static analysis will be carried out.

Author Contributions: Conceptualization, writing-review and editing, W.-h.C. and Y.T.; methodology, software, validation, formal analysis, investigation, resources, data curation, writing-original draft preparation,W.-h.C.; supervision, project administration, funding acquisition, Y.T. Both authors have read and agreed to the published version of the manuscript.

Funding: This research was funded by Grant-in-Aid for Scientific Research of Japan Society for the Promotion of Science, grant number 17H03162, Japan.

Institutional Review Board Statement: The study was approved by the Ethics Committee of the Tokyo Institute of Technology (Approval number: 2019179 and Date of approval: 28 February 2020).

Informed Consent Statement: Informed consent was obtained from all subjects involved in the study.

Acknowledgments: This research was in part supported by Grant-in-Aid for Scientific Research of Japan Society for the Promotion of Science(17H03162), and by ABE TECHNO SYSTEM Co., Ltd.

Conflicts of Interest: The authors declare no conflict of interest.

\section{References}

1. Soucacos, P. Indications and selection for digital amputation and replantation. J. Hand Surg. 2001, 26, 572-581. [CrossRef] [PubMed]

2. Bernhardt, J.; Dewey, H.; Thrift, A.; Donnan, G. Inactive and alone: physical activity within the first 14 days of acute stroke unit care. Stroke 2004, 35, 1005-1009. [CrossRef] [PubMed]

3. Who. World Health Statistics 2008; World Health Organization: Geneva, Switzerland, 2008.

4. Neumann, D.A. Kinesiology of the Musculoskeletal System: Foundations for Rehabilitation; Elsevier Health Sciences: Philadelphia, PA, USA, 2013.

5. Spartacus, V. Trapeziometacarpal Joint: A Mechanical Explanation of Total Prosthesis Failures. In Biomechanics; IntechOpen: Rijeka, Croatia, 2018.

6. Balasubramanian, S.; Klein, J.; Burdet, E. Robot-assisted rehabilitation of hand function. Curr. Opin. Neurol. 2010, 23, 661-670. [CrossRef]

7. Agarwal, P. A hand exoskeleton with series elastic actuation for rehabilitation: design, control and experimentation. Ph.D. Thesis, The University of Texas at Austin, Austin, TX, USA, 2017. 
8. Iqbal, J.; Ahmad, O.; Malik, A. HEXOSYS II-towards realization of light mass robotics for the hand. In Proceedings of the 2011 IEEE 14th International Multitopic Conference, Karachi, Pakistan, 22-24 December 2011; pp. 115-119.

9. Stergiopoulos, P.; Fuchs, P.; Laurgeau, C. Design of a 2-Finger Hand Exoskeleton for VR Grasping Simulation; Eurohaptics: Dublin, Ireland, 2003; pp. 80-93.

10. Garcia-Hernandez, N.; Sarakoglou, I.; Tsagarakis, N.; Caldwell, D. Under-Actuated Hand Exoskeleton with Novel Kinematics for Potential Use in Rehabilitation; EuroHaptics: Versailles, France, 2014; pp. 24-27.

11. Ueki, S.; Kawasaki, H.; Ito, S.; Nishimoto, Y.; Abe, M.; Aoki, T.; Ishigure, Y.; Ojika, T.; Mouri, T. Development of a hand-assist robot with multi-degrees-of-freedom for rehabilitation therapy. IEEE/ASME Trans. Mechatronics 2010, 17, 136-146. [CrossRef]

12. Wege, A.; Hommel, G. Development and control of a hand exoskeleton for rehabilitation of hand injuries. In Proceedings of the 2005 IEEE/RSJ International Conference on Intelligent Robots and Systems, Edmonton, AB, Canada, 2-6 August 2005; pp. 3046-3051.

13. Wang, J.; Li, J.; Zhang, Y.; Wang, S. Design of an exoskeleton for index finger rehabilitation. In Proceedings of the 2009 Annual International Conference of the IEEE Engineering in Medicine and Biology Society, Minneapolis, MN, USA, 3-6 September 2009; pp. 5957-5960.

14. Shields, B.L.; Main, J.A.; Peterson, S.W.; Strauss, A.M. An anthropomorphic hand exoskeleton to prevent astronaut hand fatigue during extravehicular activities. IEEE Trans. Syst. Man Cybern. Part Syst. Humans 1997, 27, 668-673. [CrossRef] [PubMed]

15. Fontana, M.; Dettori, A.; Salsedo, F.; Bergamasco, M. Mechanical design of a novel hand exoskeleton for accurate force displaying. In Proceedings of the 2009 IEEE International Conference on Robotics and Automation, Kobe, Japan, 12-17 May 2009; pp. 1704-1709.

16. Nakagawara, S.; Kajimoto, H.; Kawakami, N.; Tachi, S.; Kawabuchi, I. An encounter-type multi-fingered master hand using circuitous joints. In Proceedings of the 2005 IEEE International Conference on Robotics and Automation, Barcelona, Spain, 18-22 April 2005; pp. 2667-2672.

17. Yihun, Y.; Miklos, R.; Perez-Gracia, A.; Reinkensmeyer, D.J.; Denney, K.; Wolbrecht, E.T. Single degree-of-freedom exoskeleton mechanism design for thumb rehabilitation. In Proceedings of the 2012 Annual International Conference of the IEEE Engineering in Medicine and Biology Society, Zurich, Switzerland, 29 June-1 July 2012; pp. 1916-1920.

18. Choi, W.h.; Takeda, Y. Displacement Analysis and Design of a (2-RRU)-URR Parallel Mechanism Performing 2R1T Output Motion for Thumb Rehabilitation. Robotics 2020, 9, 67. [CrossRef]

19. Choi, W.h.; Takeda, Y. Kinematic Analysis of (2-RRU)-URR Parallel Mechanism Performing 2R1T Output Motion. In Proceedings of the IFToMM International Symposium on Robotics and Mechatronics, Taipei, Taiwan, 28-30 October 2019; pp. 114-124.

20. Choi, W.h.; Takeda, Y. Static Analysis and Actuator Selection of (2-RRU)-URR Parallel Mechanism for Thumb Rehabilitation. In Proceedings of the International Conference of IFToMM ITALY, Naples, Italy, 9-11 September 2020; pp. 608-616.

21. Batmanabane, M.; Malathi, S. Movements at the carpometacarpal and metacarpophalangeal joints of the hand and their effect on the dimensions of the articular ends of the metacarpal bones. Anat. Rec. 1985, 213, 102-110. [CrossRef] [PubMed]

22. Cooney, W.P.; Lucca, M.J.; Chao, E.; Linscheid, R. The kinesiology of the thumb trapeziometacarpal joint. J. Bone Joint Surg. Am. 1981, 63, 1371-1381. [CrossRef] [PubMed] 